\title{
Oxidative stress mediates tau-induced neurodegeneration in Drosophila
}

\author{
Dora Dias-Santagata, ${ }^{1}$ Tudor A. Fulga, ${ }^{1}$ Atanu Duttaroy, ${ }^{2}$ and Mel B. Feany ${ }^{1}$ \\ 1Department of Pathology, Brigham and Women's Hospital and Harvard Medical School, Boston, Massachusetts, USA. \\ 2Biology Department, Howard University, Washington, DC, USA.
}

\begin{abstract}
Markers of oxidative damage have been detected in brain tissue from patients with Alzheimer disease (AD) and other neurodegenerative disorders. These findings implicate oxidative injury in the neurodegenerative process, but whether oxidative stress is a cause or a consequence of neurotoxicity remains unclear. We used a Drosophila model of human tauopathies to investigate the role of oxidative stress in neurodegeneration. Genetic and pharmacological manipulation of antioxidant defense mechanisms significantly modified neurodegeneration in our model, suggesting that oxidative stress plays a causal role in neurotoxicity. We demonstrate that the JNK signaling pathway is activated in our model, which is in agreement with previous findings in AD tissue. Furthermore, we show that the extent of JNK activation correlates with the degree of tau-induced neurodegeneration. Finally, our findings suggest that oxidative stress acts not to promote tau phosphorylation, but to enhance tau-induced cell cycle activation. In summary, our study identifies oxidative stress as a causal factor in tau-induced neurodegeneration in Drosophila.
\end{abstract}

\section{Introduction}

Alzheimer disease (AD), the most prevalent form of dementia, is a devastating and ultimately fatal neurodegenerative disorder characterized by progressive loss of memory and cognition. Although early-onset familial forms of the disease have been identified, most $\mathrm{AD}$ cases are sporadic, and the major risk factor for the disease is age (1). A prominent hypothesis regarding the aging process proposes that the accumulation of oxidative damage on macromolecules is the main cause of cellular senescence (2). Oxidative stress results from an imbalance in aerobic metabolism and poses a serious threat to cellular homeostasis. Highly reactive oxygen species oxidize lipids, proteins, and DNA, leading to tissue damage and cell death. To counteract the deleterious effects of ROS, organisms have developed a number of antioxidant defense mechanisms (3).

Major support for the oxidative stress theory of aging has come from genetic studies showing that manipulation of antioxidant defense pathways affects longevity. For instance, the consecutive enzymatic activities of superoxide dismutase (SOD) and catalase are critical to eliminating superoxide anion, an extremely toxic ROS, and mutation or downregulation of these enzymes has been associated with reduced longevity in Drosophila (4-7). Conversely, increasing SOD1 or SOD2 levels can extend lifespan in Drosophila, and catalase overexpression promotes longevity in both flies and mice (8-11). Similar genetic manipulations have highlighted the importance of another line of defense in which the cyclic oxidation and reduction of thioredoxin by thioredoxin peroxidases (Tpx) and reductases (Trxr) is ultimately used for the breakdown of hydrogen peroxide (12-15).

While endogenous antioxidant pathways can protect against low levels of ROS, higher doses or persistent injury can trigger

Nonstandard abbreviations used: $\mathrm{AD}$, Alzheimer disease; APP, amyloid precursor protein; PCNA, proliferating cell nuclear antigen; $\mathrm{PH} 3$, phosphorylated histone 3; puc, puckered; SOD, superoxide dismutase; SP/TP, serine-proline/threonine-proline; $\mathrm{Tpx}$, thioredoxin peroxidase(s); Trxr, thioredoxin reductase(s).

Conflict of interest: The authors have declared that no conflict of interest exists. Citation for this article: J. Clin. Invest. 117:236-245 (2007). doi:10.1172/JCI28769. cell death. It has been well documented that oxidative stress can induce apoptosis through sustained activation of the JNK signaling pathway $(3,16)$.

In addition to its likely role in senescence, oxidative stress has also been implicated in neurodegeneration. The brain appears to be particularly sensitive to oxidative injury, and there is substantial evidence demonstrating the presence of oxidative damage in postmortem brain tissue derived from patients with age-related neurodegenerative disorders, including $\operatorname{AD}(17,18)$. Notably, markers for oxidized lipids and proteins accumulate in regions that are particularly affected by neurodegeneration $(19,20)$. Whether these markers are a mere secondary consequence of the disease or oxidative stress plays a fundamental role in neurodegeneration is a question that remains open.

Another characteristic feature of several neurodegenerative disorders, including $\mathrm{AD}$, is the ectopic expression of cell cycle markers (21-23). The presence of cell cycle markers in neurodegenerative disease tissue raised the possibility that aberrant activation of the cell cycle machinery in postmitotic neurons could be lethal and contribute to neurodegeneration. Although a connection has been established between oxidative injury and cell cycle induction, it is unclear whether oxidative stress contributes to cell cycle activation during progressive neurodegeneration (24).

In this study, we used a Drosophila model relevant to human neurodegenerative diseases, including $\mathrm{AD}$, to investigate the role of oxidative stress in neurodegeneration. A group of neurodegenerative disorders, including $\mathrm{AD}$, are characterized pathologically by the deposition of intracellular aggregates containing abnormally phosphorylated forms of the microtubule-binding protein tau (25). These disorders are often termed tamopathies. Mutations in the tau gene itself have been identified in familial forms of frontotemporal dementias (26), demonstrating that abnormalities in tau can directly mediate neurodegeneration. Consistent with this hypothesis, expression of wild-type and mutant forms of human tau in the Drosophila nervous system results in a neurodegenerative phenotype that recapitulates several characteristics of human tauopathies (27, 28). This model provides an in vivo system useful for dissecting the 

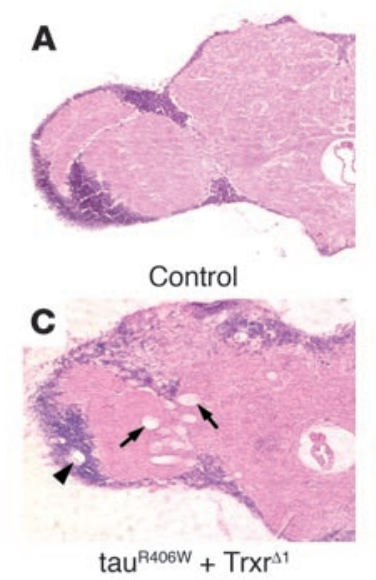

$\operatorname{tau}^{\mathrm{R} 406 \mathrm{~W}}+\operatorname{Trxr}^{\Delta 1}$

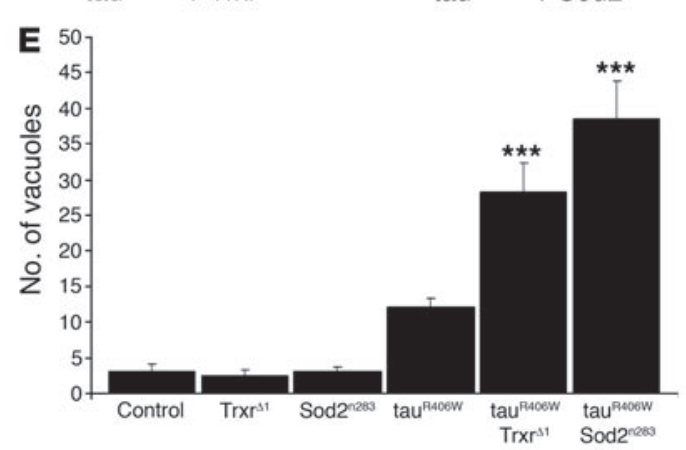

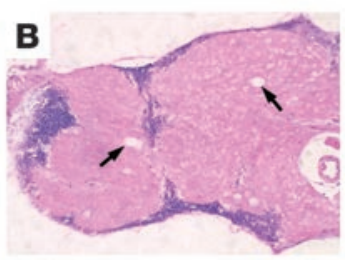

tau ${ }^{\text {R406w }}$

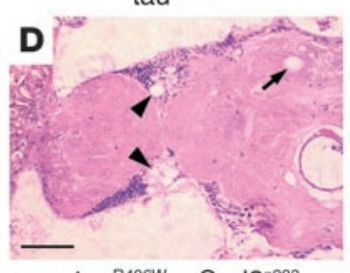

$\operatorname{tau}^{\mathrm{P} 406 \mathrm{~W}}+$ Sod2 $2^{\mathrm{n} 283}$

\section{(a)}

\section{Figure 1}

Partial inactivation of antioxidant pathways enhances neurodegeneration in a Drosophila tauopathy model. (A-D) Frontal brain sections of 10-day-old flies were stained with H\&E. Neurodegeneration was not detected in control flies (A) but was evident in transgenic flies expressing tau ${ }^{\mathrm{R} 406 \mathrm{~W}}$ (B). Tau-induced toxicity was enhanced by removing 1 functional copy of Trxr in tauR406W transgenic flies heterozygous for the $\operatorname{Tr} \mathrm{r}^{\Delta 1}$-null allele (C) and by reducing gene dosage of mitochondrial Sod2 in tauR406W transgenic flies heterozygous for a null allele $\left(\right.$ Sod2 $\left.{ }^{\text {2283 }}\right)$ (D). Vacuolar degeneration was observed in the neuropil (arrows) and in the cortex (arrowheads). Scale bar: $20 \mu \mathrm{m}$. (E) Tauinduced toxicity was significantly enhanced by genetic downregulation of $\operatorname{Trxr}\left({ }^{* \star *} P<0.001\right)$ and Sod2 $\left({ }^{* * *} P<0.001\right)$ activities. Neurodegeneration was assessed by quantification of vacuoles with diameter greater than $3 \mu \mathrm{m}$ in the brains of 10-day-old flies. Data points represent the mean \pm SEM. In Figures 1, 2, and 7, genotypes are as follows: control, elav-GAL4/+; Trxr ${ }^{\Delta 1}$, elav-GAL4/Trxr ${ }^{\Delta 1}$; Sod2 ${ }^{\mathrm{n} 283}$, elavGAL4/+, Sod2n283/+; tau ${ }^{\mathrm{R} 406 \mathrm{~W}}$, elav-GAL4/+, UAS-tau ${ }^{\mathrm{R} 406 \mathrm{~W} /+}$; tau ${ }^{\mathrm{R} 406 \mathrm{~W}}$ $+\operatorname{Trxr}^{\Delta 1}$, elav-GAL4/Trxr ${ }^{\Delta 1}$, UAS-tau ${ }^{R 406 \mathrm{~W} /+}$; and tau ${ }^{\mathrm{R} 406 \mathrm{~W}}+\mathrm{Sod}^{\mathrm{n} 283}$, elav-GAL4/+, Sod2n283/+, UAS-tauR406W/+. molecular mechanisms underlying neurodegeneration, and we have used it to address the role of oxidative stress in neuronal cell death. We have employed genetic and pharmacological approaches to modify the phenotype of transgenic flies that express a diseaserelated mutant form of human tau $\left(\operatorname{tau}^{\mathrm{R} 406 \mathrm{~W}}\right)$ in a panneuronal pattern. Our results strongly suggest that oxidative stress plays a causal role in tau-induced neurodegeneration. We also found that tau-induced toxicity is accompanied by activation of the JNK signaling pathway. Interestingly, the extent of JNK pathway activation directly correlated with neurotoxicity levels. Further analysis indicated that oxidative stress did not promote tau phosphorylation in our model and suggested that oxidative injury may trigger apoptosis by intensifying tau-mediated cell cycle activation.

\section{Results}

Oxidative stress mediates neurotoxicity in a Drosophila tanopathy model. To determine whether oxidative damage plays an active role in neurodegeneration, we first tested whether genetic downregulation of antioxidant defense pathways would enhance tau-induced toxicity. Overexpression of mutant tau ( $\left.\operatorname{tau}^{\mathrm{R} 406 \mathrm{~W}}\right)$ in the fly brain results in a progressive neurodegenerative phenotype characterized by histological abnormalities, including degeneration of neurons in the cortex and neuropil vacuolization (27), that can be visualized in standard histological preparations. The cell bodies of neurons and glia occupy the outer cortical layer of the brain, which is basophilic and can be distinguished after H\&E staining by a dark purple color. Processes of neurons and glia are located in the inner neuropil region, which is eosinophilic and stains pink or light purple following H\&E processing (Figure 1, A-D, and Supplemental Figures 1, A-F, and 2, A and B; supplemental material available online with this article; doi:10.1172/JCI28769DS1). The brains of control flies were well preserved at 10 days of age (Figure $1 \mathrm{~A})$, but degenerative changes were apparent in both the cortex and the neuropil of $\mathrm{tau}^{\mathrm{R} 406 \mathrm{~W}}$ transgenic flies by day 10 (Figure $1 \mathrm{~B}$ and Supplemental Figure 1, A and B) (27). Heterozygosity for a $\operatorname{Tr} x r$-null allele $\left(\operatorname{Tr} x r^{\Delta 1}\right)$ that removes both the mitochondrial and cytoplasmic forms of the enzyme (15) enhanced tau toxicity in the brain as evidenced by an increase in cortex and neuropil vacuolization (Figure 1C and Supplemental Figure 1, C and D). Similarly, reduced gene dosage of mitochondrial Sod2 in tau transgenic flies heterozygous for a null allele ( $\left.\operatorname{Sod} 2^{n 283}\right)$ (4) also enhanced tauinduced neurodegeneration (Figure 1D and Supplemental Figure 1, E and F). Quantification of the total number of vacuoles present in the cortex and neuropil of 10-day-old transgenic animals and controls revealed that brain vacuolization in tau ${ }^{\mathrm{R} 406 \mathrm{~W}}$-expressing flies heterozygous for $\operatorname{Tr} \mathrm{r}^{\Delta 1}$ or for $\operatorname{Sod}^{n 283}$ was significantly increased by greater than 2-fold and 3-fold, respectively, compared with that of $\mathrm{tau}^{\mathrm{R} 406 \mathrm{~W}}$ transgenic animals (Figure 1E).

Tau-induced neurodegeneration occurs by an apoptotic cell death mechanism in our model (27-29). While no TUNEL-positive cells were detected in the brains of 10-day-old controls (Figure 2A), modest numbers of apoptotic nuclei were present in age-matched tau transgenic flies (Figure 2B) (27). Significantly increased numbers of TUNEL-positive cells were present in tau transgenic animals heterozygous for $\operatorname{Tr} r^{\Delta 1}$ (Figure 2C) or for $\operatorname{Sod}^{\text {n283 }}$ (Figure 2D). Quantitative analysis revealed a greater than 2-fold increase in the number of apoptotic cells compared with that of $\mathrm{tau}^{\mathrm{R} 406 \mathrm{~W}}$ transgenics (Figure 2E). In contrast, heterozygosity for either $\operatorname{Tr}_{x} r^{\Delta 1}$ or $\operatorname{Sod} 2^{n 283}$ in the absence of $\mathrm{tau}^{\mathrm{R} 406 \mathrm{~W}}$ expression was not associated with neurodegeneration. In fact, $\operatorname{Sod}^{n 283}$ and $\operatorname{Tr} x r^{\Delta 1}$ heterozygous flies were indistinguishable from controls at day 10 , with respect to gross brain morphology (compare Supplemental 

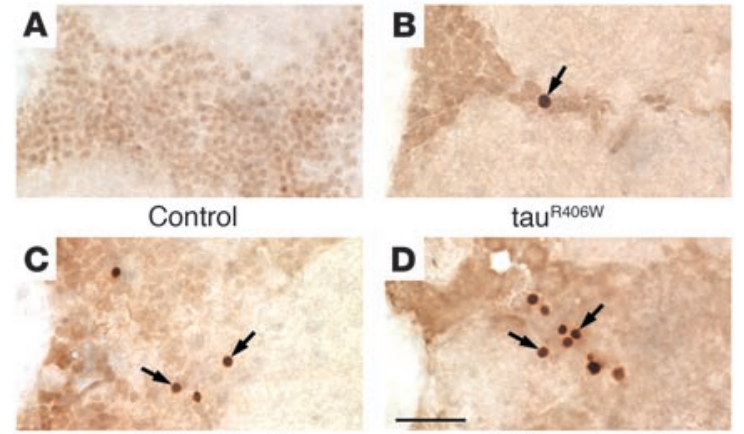

$\operatorname{tau}^{\mathrm{R} 406 \mathrm{~W}}+\operatorname{Trxr}^{\Delta 1}$

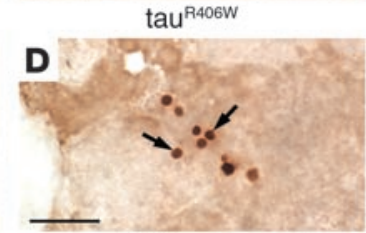

$\operatorname{tau}^{\mathrm{R} 406 \mathrm{~W}}+\mathrm{Sod}^{\mathrm{n} 283}$
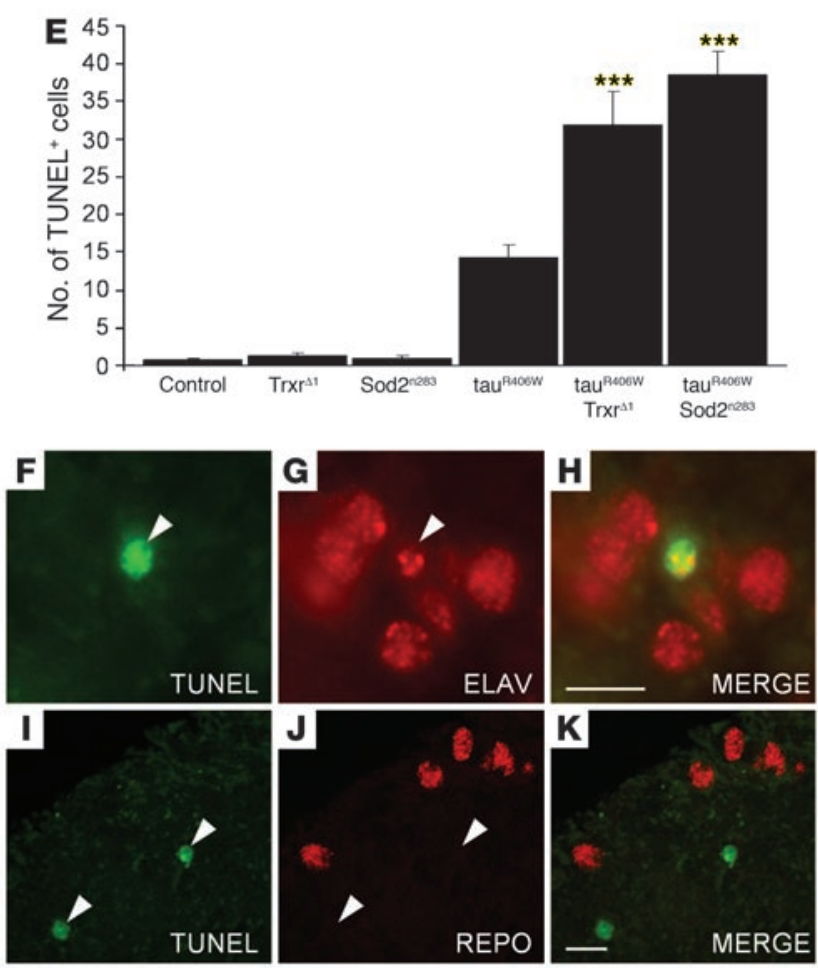

Figure 2, A and B, with Figure 1A), total number of vacuoles (Figure 1E), and neuronal cell death (Figure 2E). Notably, in older flies heterozygous for $\operatorname{Sod} 2^{n 283}$ or for $\operatorname{Tr} x r^{\Delta 1}$, brain vacuolization and cell death were still comparable to that of age-matched control flies (Supplemental Figure 2, C and D).

To identify the nature of the apoptotic cells identified in tau ${ }^{\mathrm{R} 406 \mathrm{~W}}$-expressing flies, brain sections of 10-day-old transgenic flies were costained with TUNEL and with antibodies recognizing either a neuronal cell marker (elav) or a glial cell marker (repo) (Figure 2, F-K). TUNEL-positive cells also expressed elav (Figure 2 , F-H). In contrast, there was no evidence of TUNEL-positive cells colocalizing with repo-expressing cells (Figure 2, I-K). These observations suggest that the large majority of apoptotic cells identified in the brains of tau transgenic flies are neurons, suggestive of a cell-autonomous mechanism of tau-induced cell death.

Taken together, histological analysis and quantification of neuronal apoptosis demonstrated that the downregulation of antioxidant defense pathways significantly enhanced tau neurotoxicity. Moreover, the absence of neurodegeneration in Sod2 ${ }^{\text {n283 }}$ and $\operatorname{Tr} r^{\Delta 1}$ heterozygous flies suggested that this enhancement

\section{Figure 2}

Downregulation of Sod2 or of Trxr antioxidant activities increases neuronal cell death in a Drosophila tauopathy model. (A-D) TUNEL assay in 10-day-old transgenic fly brains. Apoptotic cells, absent in control animals $(\mathbf{A})$, were identified by TUNEL staining (arrows) in the brains of tau ${ }^{\mathrm{R} 406 \mathrm{~W}}$ transgenic flies (B) and were found to be more abundant in tauR406W flies heterozygous for $\operatorname{Trxr}^{\Delta 1}$ (C) and for Sod2 ${ }^{\text {n283 }}$ (D). Scale bar: $10 \mu \mathrm{m}$. (E) Neurodegeneration was significantly enhanced by partial inactivation of $\operatorname{Trxr}\left({ }^{* \star *} P<0.001\right)$ and Sod2 $\left({ }^{* \star *} P<0.001\right)$ activities. The number of TUNEL-positive cells in the brains of 10-dayold tauR406W-expressing flies was compared with that in age-matched controls, in Sod2 ${ }^{\mathrm{n} 283}$ and $\operatorname{Tr}_{\mathrm{r}} \mathrm{r}^{\Delta 1}$ heterozygous animals, and in tau ${ }^{\mathrm{R} 406 \mathrm{~W}}$ flies heterozygous for either Sod2 ${ }^{n 283}$ or $\operatorname{Tr} \mathrm{rr}^{\Delta 1}$. Data points represent the mean \pm SEM. $(\mathbf{F}-\mathbf{K})$ Tau-induced toxicity was associated with neuronal cell death. TUNEL-positive cells (green fluorescence) were found to coexpress the neuronal cell marker elav (red fluorescence) $(\mathbf{F}-\mathbf{H})$ and did not colocalize with repo-positive glial cells (red fluorescence) (I-K). Arrowheads mark the location of TUNEL-positive cells. Scale bars: $5 \mu \mathrm{m}$.

was not the added effect of 2 toxicities but was instead the result of synergistic effects between tau and increased oxidative stress.

To test whether decreasing oxidative stress can rescue neurodegeneration, we coexpressed $\mathrm{tau}^{\mathrm{R} 406 \mathrm{~W}}$ with Sod2 (30), which led to a $50 \%$ decrease in the number of TUNEL-positive cells found in tau transgenics (Figure 3A). In addition, coexpression of tau ${ }^{\mathrm{R} 406 \mathrm{~W}}$ with Tpx1 (14) resulted in a greater than $60 \%$ reduction of brain apoptosis compared with tau alone (Figure $3 \mathrm{~A})$. To determine whether the tau neurodegenerative phenotype is amenable to pharmacological intervention, we fed tau ${ }^{\mathrm{R} 406 \mathrm{~W}}$-expressing flies and control flies for 10 days with 2 different levels of vitamin E. We used $\alpha$-tocopherol, a vitamin $\mathrm{E}$ isoform that is the most powerful naturally occurring antioxidant known to date (31). Control cohorts within each genotype were fed with soybean oil solvent alone. Neurodegeneration associated with expression of $\mathrm{tau}^{\mathrm{R} 406 \mathrm{~W}}$ was significantly suppressed by treatment of adult flies with $0.5 \mathrm{mM}$ and $1.5 \mathrm{mM}$ of vitamin $\mathrm{E}$, as evidenced by $30 \%$ and $50 \%$ declines in the number of TUNELpositive cells, respectively, when compared with tau transgenic flies fed with solvent only (Figure 3B). Treatment with vitamin E did not influence cell death in control animals (Figure 3B). In addition, Western blot analysis revealed that the levels of tau protein were not altered by feeding flies with vitamin $\mathrm{E}$ (data not shown). This result indicates that the decrease in toxicity observed in drug-fed tau $^{\mathrm{R} 406 \mathrm{~W}}$ flies is not caused by a downregulation of tau transgene expression and suggests a neuroprotective role for vitamin E. Overexpression of antioxidant enzymes and treatment with vitamin $\mathrm{E}$ were not sufficient to completely reduce neuronal cell death to the levels observed in control flies, but they significantly rescued neurotoxicity in tau ${ }^{\mathrm{R} 406 \mathrm{~W}}$ transgenic animals (Figure 3, A and B). Taken together, these observations suggest that tau-induced neurodegeneration is, at least in part, mediated by oxidative damage.

To determine whether tau-expressing flies have altered susceptibility to oxidative injury, we treated tau ${ }^{\mathrm{R} 406 \mathrm{~W}}$ transgenic flies and controls with the drug paraquat, a widely used free radical generator that can increase ROS levels in vivo (32). Administration of a total of $30 \mathrm{mM}$ paraquat over 48 hours induced $30 \%$ lethality in control flies (Figure 4). The tau ${ }^{\mathrm{R} 406 \mathrm{~W}}$ transgenic animals were significantly more sensitive to paraquat than controls, exhibiting a $70 \%$ mortality rate (Figure 4 ). There was no significant lethality associated with either tau transgenics or control flies after 48 hours of feeding on drug-free food (Figure 4). 
A
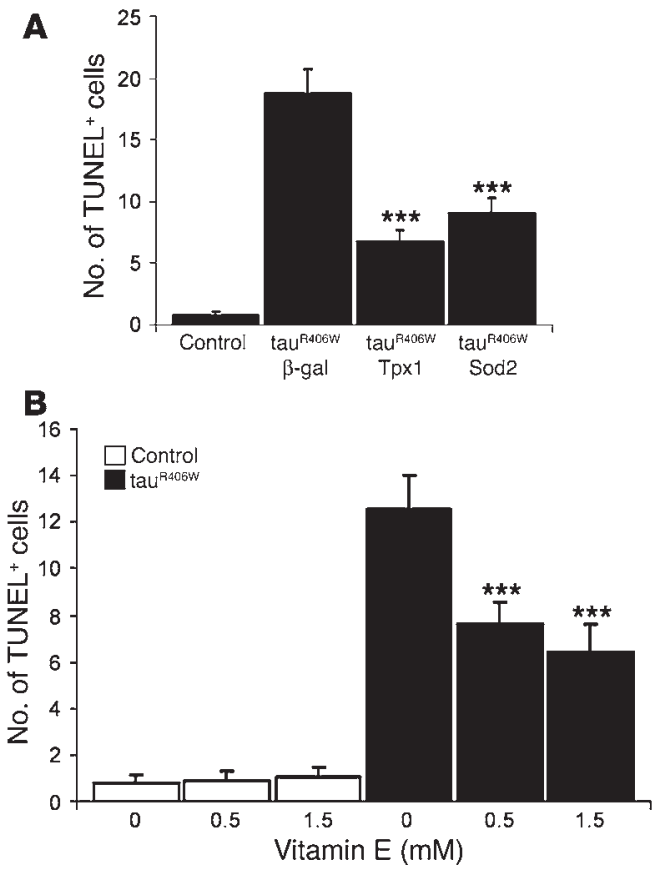

Figure 3

Genetic and pharmacological upregulation of antioxidant defenses suppresses tauinduced toxicity in vivo. (A) Neurodegeneration was significantly reduced by coexpressing tauR406W with Tpx1 $\left.{ }^{* * *} P<0.001\right)$ or with Sod2 $\left.{ }^{* * *} P<0.001\right)$. Neuronal cell death in tau ${ }^{\mathrm{R} 406 \mathrm{~W}}$ transgenic flies that have increased expression of $\operatorname{Tpx} 1(P<0.05)$ or of Sod2 $(P<0.01)$ was still significantly higher than in controls, indicating that tauinduced neurotoxicity was not completely rescued by these increased antioxidant activities. Quantification of TUNEL-positive cells in the brains of 10-day-old flies was used to evaluate neurotoxicity in controls and in tauR406W transgenics coexpressing either $\beta$-gal (control transgene), Tpx1, or Sod2. Genotypes are as follows: control, elav-GAL4/+; tau ${ }^{\mathrm{R} 406 \mathrm{~W}}+\beta$-gal, elav-GAL4/+, UAS-lacZ/+, UAS-tau $8406 \mathrm{~W} /+$; $\operatorname{tau}^{\mathrm{R} 406 \mathrm{~W}}+$ Tpx1, elav-GAL4/+, UAS-Tpx1/+, UAS-tau ${ }^{R 406 W /+; ~ a n d ~ t a u ~ R 406 W ~+~ S o d 2, ~ e l a v-G A L 4 /+, ~}$ UAS-Sod241/+, UAS-tauR406W/+. (B) Administration of vitamin $E$ for 10 days after eclosion suppressed tau-induced neurodegeneration. TUNEL assay quantification measured apoptosis in the brains of tau ${ }^{\mathrm{R} 406 \mathrm{~W}}$-expressing flies and control flies fed with soybean oil solvent (control) or with the indicated doses of vitamin E. Tau-induced neurodegeneration was significantly suppressed in tauR406W transgenic flies by feeding with $0.5 \mathrm{mM}\left({ }^{* * *} P<0.001\right)$ or $1.5 \mathrm{mM}\left({ }^{* * *} P<0.001\right)$ of vitamin $\mathrm{E}$ compared with feeding with solvent only. Treatment of tauR406W transgenic flies with vitamin $E$ did not completely abolish neuronal cell death, since toxicity was still significantly higher $(P<0.001)$ than in control flies. Error bars indicate \pm SEM. Genotypes are as follows: control, elav-GAL4/+; and tau R406W, elav-GAL4/+, UAS-tau ${ }^{R 406 W /+.}$
In summary, we found that both genetic and pharmacological manipulation of antioxidant defense mechanisms can modify tau toxicity in vivo. In particular, the fact that oxidative stress suppressors had a protective effect on neurotoxicity strongly suggests that oxidative damage plays a causal role in tau-induced neurodegeneration.

Oxidative damage does not act by altering tau phosphorylation. One of the major pathological features of $\mathrm{AD}$ and other tauopathies is the accumulation of abnormally phosphorylated forms of tau in the brains of affected individuals (25). Phosphorylation of tau at serines and threonines that are followed by a proline residue (SP/TP sites) is particularly characteristic of neurodegenerative disease tissue (33). Antibodies AT8, AT100, AT180, TG3, and PHF1, which recognize tau phosphorylated at SP/TP sites, specifically reacted with the brains of tau ${ }^{\mathrm{R} 406 \mathrm{~W}}$-expressing flies but not with those of control flies (Figure 5A) (27-29). Expression of $\mathrm{tau}^{\mathrm{R} 406 \mathrm{~W}}$ in flies heterozygous for the $\operatorname{Tr} \mathrm{x} \mathrm{r}^{\Delta 1}$-null mutation or for

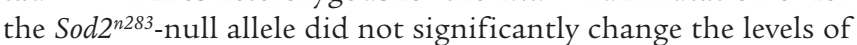
phosphorylated tau in the brains of transgenic flies. The tested animals also expressed equivalent levels of total tau and of Tau1, which recognizes an unphosphorylated form of tau (Figure 5A). Our results indicate that, despite enhancing neurodegeneration, increased oxidative stress did not appear to modify tau phosphorylation. This observation suggested that increased oxidative damage does not act to promote tau phosphorylation in our model.

The fact that aberrantly phosphorylated tau accumulates during neurodegeneration suggests that phosphorylation may contribute to toxicity. Support for this hypothesis has recently been provided by a number of studies $(28,29,34,35)$. Moreover, in our laboratory, expression of mutant tau constructs in Drosophila revealed a direct correlation between phosphorylation and tau-induced degeneration (Steinhilb et al., unpublished observations; ref. 35). In one of these constructs, designated tau ${ }^{\mathrm{E} 14}, 14$ disease-associated SP/TP phosphorylation sites were mutated to glutamate to mimic phosphorylation. This mutant tau construct is highly toxic (35). To further test the hypothesis that oxidative stress does not act via altering tau phosphorylation, we determined whether the toxicity associated with tau ${ }^{\mathrm{E} 14}$ could be enhanced by removing 1 copy of the Trxr or the Sod2 genes. Reducing the levels of these antioxidant enzymes in flies expressing tau ${ }^{\mathrm{E} 14}$ resulted in a severe decrease in viability. When compared with the expected eclosion frequency obtained for control flies expressing tau ${ }^{\mathrm{E} 14}$, the frequency of eclosion for tau ${ }^{\mathrm{E} 14}$ transgenic flies heterozygous for $\operatorname{Tr} x r^{\Delta 1}$ or for Sod $2^{n 283}$ was reduced by $36 \%$ and by $66 \%$, respectively. Rare escapers also had reduced adult viability, with the majority of these animals dying before 10 days after eclosion (data not shown). Higher levels of oxidative stress resulted in increased neuronal cell death for tau ${ }^{\mathrm{E} 14}$ transgenic flies (Figure 5B). Quantification of apoptotic cells in the brains of 5-day-old transgenic flies revealed that expres-

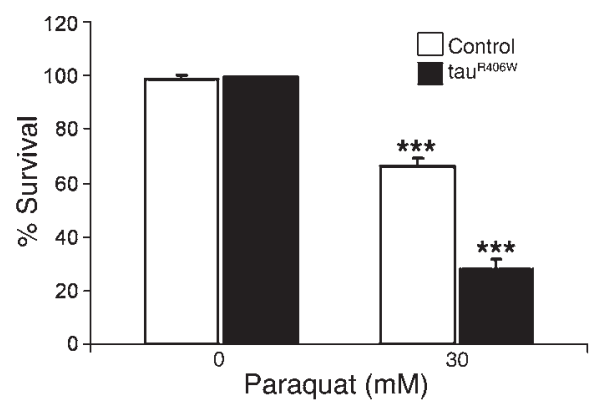

Figure 4

TauR406W transgenic flies are more sensitive to oxidative injury than age-matched controls. One-day-old flies were exposed to no drug or to $30 \mathrm{mM}$ paraquat, and survival was scored after 48 hours. Compared with use of drug-free medium, administration of paraquat resulted in significant lethality for control flies $\left({ }^{* * *} P<0.001\right)$ and for tauR406W

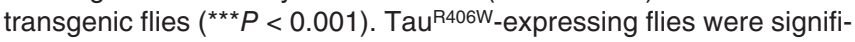
cantly more sensitive to $30 \mathrm{mM}$ paraquat $(P<0.001)$ than controls. Genotypes are as follows: control, elav-GAL4/+; and tau ${ }^{\mathrm{R} 406 \mathrm{~W}}$, elavGAL4/+, UAS-tauR406W/+. 
sion of tau ${ }^{\mathrm{E} 14}$ in flies heterozygous for $\operatorname{Tr} \mathrm{r}^{\Delta 1}$ resulted in a 2-fold increase in the number of TUNEL-positive neurons compared with that in transgenic flies expressing tau ${ }^{\mathrm{E} 14}$ alone (Figure 5B). Expression of $\mathrm{tau}^{\mathrm{E} 14}$ in animals heterozygous for $\operatorname{Sod} 2^{\text {n283 }}$ led to a 2.5 -fold increase in brain apoptosis compared with that in tau ${ }^{\mathrm{E} 14}$ transgenics (Figure 5B). Thus, neurodegeneration mediated by tau ${ }^{\mathrm{E} 14}$ was significantly enhanced by a genetic increase in oxidative stress levels. Taken together, our results strongly suggest that oxidative damage does not act by influencing tau phosphorylation.

JNK pathway activation correlates with tau-induced neurotoxicity. The induction of stress-activated protein kinase cascades, such as the JNK signaling pathway, is one of the best-characterized responses to oxidative damage $(3,36)$. To determine whether JNK pathway activation correlated with tau-induced neurodegeneration in our model, we first used TUNEL quantification to assess neuronal cell death in control flies and in flies that expressed different forms of tau (Figure 6A). This analysis confirmed that wild-type tau (tau ${ }^{\mathrm{WT}}$ ) is moderately toxic, the disease-associated mutant tau ${ }^{\mathrm{R} 406 \mathrm{~W}}$ has increased toxicity, and the pseudophosphorylated mutant tau ${ }^{\mathrm{E} 14}$ is even more toxic (Figure 6A) $(27,35)$. To measure JNK pathway activation, we immunostained the brains of 10-day-old adult flies with an antibody that recognizes activated JNK protein. Minimal immunoreactivity for active JNK was present in the cortex of control flies (Figure 6B) while active JNK accumulated in cortical areas, showing histological evidence of neurodegeneration in all the tau transgenic flies ( $\operatorname{tau}^{\mathrm{E} 14}$ is depicted in Figure 6C). We additionally employed the puckered-lacZ (puc-lacZ) reporter system, which has been commonly used to monitor JNK pathway activation in Drosophila. The puc gene encodes a phosphatase that inactivates JNK and other MAPK family members and is a well-established transcriptional target of JNK signaling in Drosophila (37). Brains from transgenic flies carrying the puc-lac $Z$ reporter alone or with various tau transgenes were immunostained at 10 days after eclosion with an antibody against $\beta$-gal. Reporter activity was minimal in control flies (Figure 6, D and F) while numerous cortical cells were $\beta$-gal-positive in tau transgenic animals (Figure 6, E and F). Quantification of JNK activity in the cortex of transgenic animals revealed that the number of $\beta$-gal-positive neurons was increased by over 2-fold for tau ${ }^{\mathrm{WT}}, 6$-fold for tau ${ }^{\mathrm{R} 406 \mathrm{~W}}$, and 9-fold for tau ${ }^{\mathrm{E} 14}$ flies compared with control flies that did not express human tau (Figure 6F). These results showed a close correlation between JNK pathway activation and the degree of neurotoxicity associated with different tau transgenes. Furthermore, $\beta$-gal-positive staining was identified in cells that also expressed the neuronal cell marker elav (Figure 6, G-I) but not in repo-positive glial cells (Figure 6, $\mathrm{J}-\mathrm{L}$ ), suggesting that in our tauopathy model, JNK activation occurs mostly in neurons.

Oxidative stress enhances cell cycle activation. We have recently shown that cell cycle activation mediates neurodegeneration in tau transgenic flies (35). To determine whether oxidative stress promotes tau-induced neurodegeneration by activating the cell cycle, brains of tau ${ }^{\mathrm{R} 406 \mathrm{~W}}$-expressing flies aged for 10 days were immunostained with an antibody against the proliferating cell nuclear antigen (PCNA), an S-phase marker abnormally reexpressed in human degenerative disease tissue (22) as well as in tau transgenic flies (35). No PCNA immunostaining was detected in control animals (Figure $7 \mathrm{~A})$, but multiple foci were present in tau ${ }^{\mathrm{R} 406 \mathrm{~W}}$ transgenic brains (Figure 7B). The tau ${ }^{\mathrm{R} 406 \mathrm{~W}}$-expressing flies haploinsufficient for Trxr (Figure 7C) or Sod2 (Figure 7D) exhibited substantially higher levels of PCNA staining in their brains than transgenic animals expressing tau ${ }^{\mathrm{R} 406 \mathrm{~W}}$ alone (Figure $7 \mathrm{~B}$ ). PCNA staining accumulated in areas of neurodegeneration (Figure 7, B-D). Quantification of PCNA immunostaining revealed that the number of PCNA-positive foci found in the brains of tau ${ }^{\mathrm{R} 406 \mathrm{~W}}$ transgenic flies increased by more than 2 -fold when tau ${ }^{\mathrm{R} 406 \mathrm{~W}}$ was expressed in a Trxr heterozygous or in a Sod2 heterozygous background (Figure $7 \mathrm{E})$. Our results showed that oxidative stress can promote cell cycle activation and apoptosis in the brains of tau transgenic flies, thereby supporting the hypothesis that oxidative damage may promote neurodegeneration by enhancing tau-induced cell cycle activation. In addition, there were no signs of cell cycle activation in flies heterozygous for either $\operatorname{Tr} x r^{\Delta 1}$ or $\operatorname{Sod} 2^{n 283}$, which were indistinguishable from age-matched controls as determined by PCNA immunostaining (Figure 7E). This observation suggested that, in our model, enhancement of cell cycle activation induced by oxidative damage resulted from a synergistic effect.

Phosphorylated histone 3 ( $\mathrm{PH} 3$ ) is another cell cycle marker found to be aberrantly expressed in $\mathrm{AD}(21)$ and in tau transgenic flies but not in controls (35). To determine which cell type undergoes cell cycle induction in our model, brain sections from 10-dayold tau ${ }^{\mathrm{R} 406 \mathrm{~W}}$ transgenic flies were costained with antibodies specific for PH3 and either the neuronal marker elav or the glial marker repo. This analysis revealed that $\mathrm{PH} 3$-positive cells also expressed

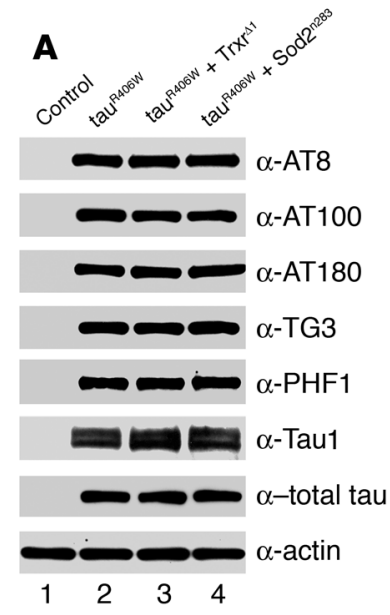

B

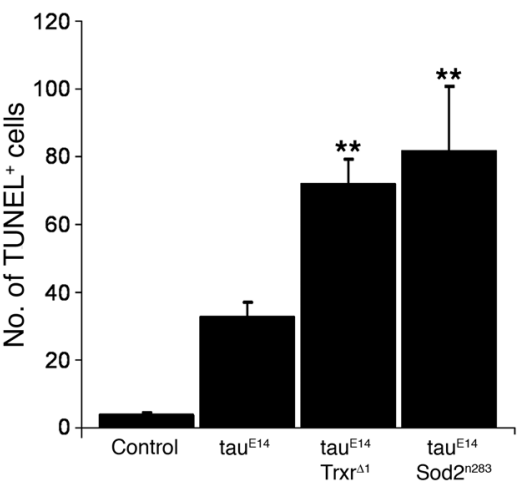

\section{Figure 5}

Oxidative stress does not act by altering tau phosphorylation. (A) Increased oxidative stress did not clearly modify tau phosphorylation. Equivalent amounts of fly head extracts (day 10) were analyzed by Western immunoblotting using the indicated antibodies. Genotypes for each column are as follows: control, elav-GAL4/+; tauR406W, elav-GAL4/+, UAS-tauR406W/+; tauR406W $+\operatorname{Trxr}^{\Delta 1}$, elav-GAL4/Trxr ${ }^{\Delta 1}$, UAS-tau ${ }^{R 406 \mathrm{~W} /+}$; and tau ${ }^{\mathrm{R} 406 \mathrm{~W}}+\mathrm{Sod}^{\mathrm{n} 283}$, elavGAL4/+, Sod2n283/+, UAS-tauR406W/+. (B) Genetic downregulation of antioxidant enzymes enhanced neurotoxicity in transgenic flies expressing a pseudophosphorylated mutant form of tau. Compared with that in flies expressing tau $^{\mathrm{E} 14}$, neurodegeneration was significantly increased in tau ${ }^{\mathrm{E} 14}$ transgenic animals heterozygous for $\operatorname{Tr} r^{\Delta 1}\left({ }^{* *} P<0.01\right.$ ) or for Sod $2^{n 283}\left({ }^{* *} P<0.01\right.$ ). Quantification of TUNEL-positive cells in the brains of 5-day-old transgenic flies was used to evaluate neurotoxicity levels in transgenic animals. Genotypes are as follows: control, elav-GAL4/+, UAS-tau'WT/+; tauE14, elav-GAL4/+, UAS-tau ${ }^{E 14(22) /+;} \operatorname{tau}^{E 14}+\operatorname{Trxr}^{\Delta 1}$, elav-GAL4/Trxr ${ }^{\Delta 1}$, UAS-tau ${ }^{E 14(22) /+;}$ and tauE14 + Sod2 ${ }^{\text {n283 }}$, elav-GAL4/+, Sod2n283/+, UAS-tauE14(22)/+. 

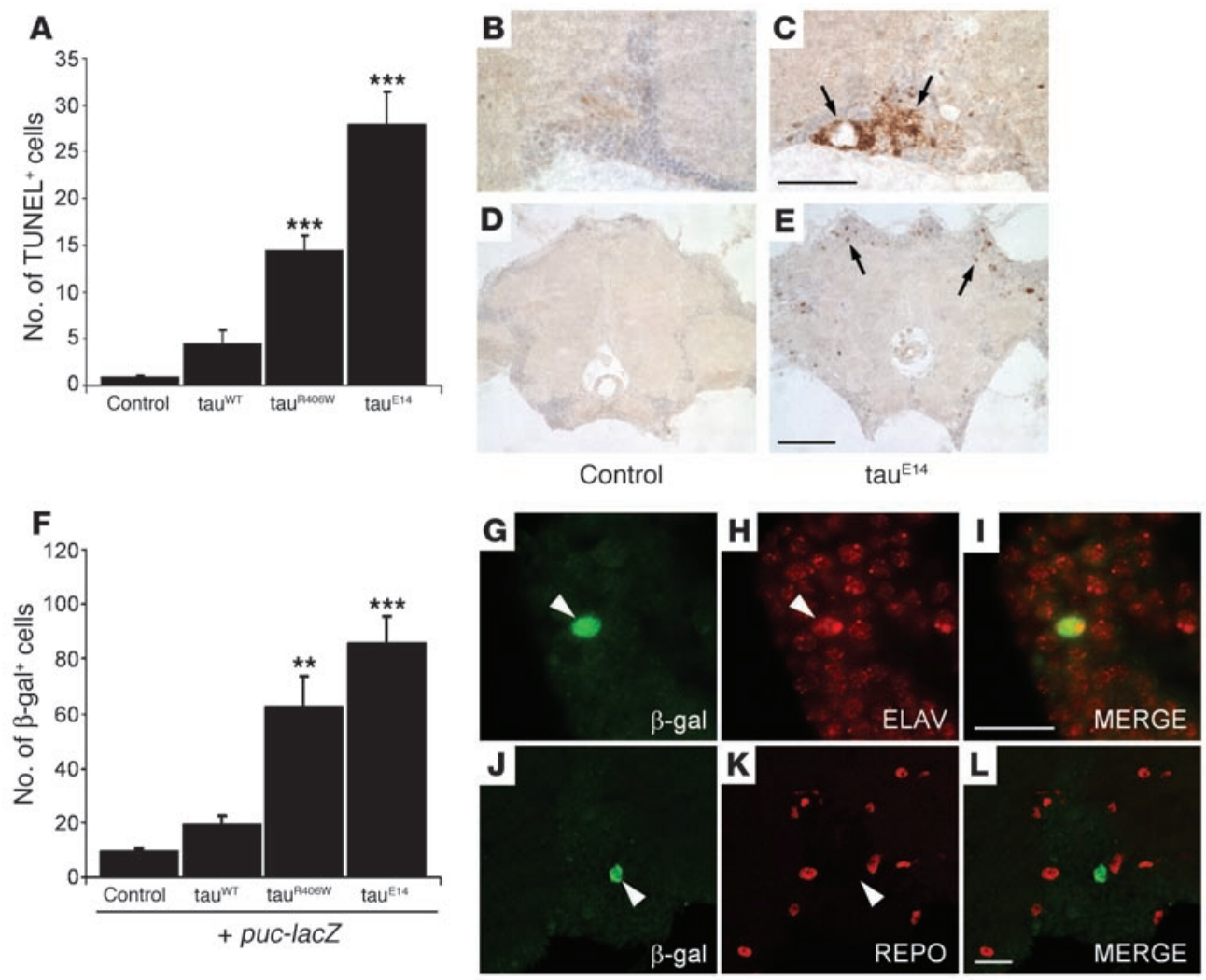

\section{Figure 6}

Tau-induced toxicity is accompanied by JNK pathway activation. (A) Quantification of TUNEL-positive neurons in the brains of 10-day-old flies expressing different tau isoforms revealed significantly higher neurotoxicity in tau ${ }^{\mathrm{R} 406 \mathrm{~W}}\left({ }^{\star \star \star} P<0.001\right)$ and $\operatorname{tau}^{\mathrm{E} 14}\left({ }^{\star \star \star} P<0.001\right)$ transgenic flies compared with controls and tauWT-expressing flies. Neurodegeneration was significantly higher in tauE14- than in tauR406W-expressing flies $(P<0.001)$. (B and $\mathbf{C})$ The brains of 10-day-old control $(\mathbf{B})$ and tauE14-expressing $(\mathbf{C})$ flies were immunostained with a phosphorylated (active) JNK-specific antibody, which accumulated in areas of neurodegeneration (arrows). Scale bar: $20 \mu \mathrm{m}$. Genotypes (A-C) are as follows: control, elav-GAL4/+; tau'WT, elav-GAL4/+, UAS-tauWT/+; tauR406W, elav-GAL4/+, UAS-tau ${ }^{\mathrm{R} 406 \mathrm{~W} /+ \text {; }}$ and tau ${ }^{\mathrm{E} 14}$, elav-GAL4/+, UAS-tau $144(26) /+$. (D and E) puc-lacZ gene expression revealed JNK pathway activation in tau transgenic flies. Brains of transgenic flies expressing the puc-lac $Z$ transgene alone (D) or in combination with tau ${ }^{\mathrm{E} 14}$ (E) were immunostained for $\beta$-gal (arrows). Scale bar: $20 \mu \mathrm{m}$. (F) JNK pathway activation correlated with the extent of neurotoxicity induced by different tau transgenes. Quantification of $\beta$-gal-positive cells in 10 -day-old puc-lac $Z$ transgenic fly brains identified significant increases in JNK pathway activation in animals that also expressed tau ${ }^{\mathrm{R} 406 \mathrm{~W}}\left({ }^{* *} P<0.01\right)$ or tauE14 $\left({ }^{* * *} P<0.001\right)$ compared with tauWT-expressing flies or controls. (G-L) JNK pathway activation was detected in neurons. $\beta$-gal-positive cells (green fluorescence) often coexpressed the neuronal cell marker elav (red fluorescence) (G-I) and did not colocalize with repo-positive glia (red fluorescence) (J-L). Arrowheads mark the locations of $\beta$-gal-positive cells. Scale bars: $10 \mu \mathrm{m}$. Genotypes (D-L) are as follows: control, elav-GAL4/+, puc-lacZ/+; tauwT,

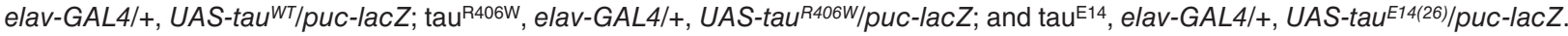

elav (Figure 7, F-H) but not repo (Figure 7, I-K). Although it is possible that some glial cells experience cell cycle reentry, these findings suggest that aberrant cell cycle reentry in our tauopathy model is, for the most part, a neuronal event.

\section{Discussion}

In this study, we provide substantial in vivo evidence supporting a causative role for oxidative stress in tau-induced neurodegeneration. It is well known that oxidative damage markers accumulate in tissue samples derived from patients with neurodegenerative disorders, such as AD and tauopathies $(19,20,38)$, but whether this is a result of the disease process or whether oxidative stress actively induces neurodegeneration is still debated (17). Using a Drosophila model of human tauopathy, we show that genetic and pharmacological upregulation of antioxidant defenses significantly rescues neurotoxicity in the brains of tau transgenic flies
(Figure 3). Conversely, genetic inactivation of antioxidant mechanisms enhances tau-induced neurodegeneration (Figures 1 and 2). These observations strongly implicate oxidative stress as a critical mediator of tau-induced neurotoxicity.

Despite significant effort, we were unable to detect clear changes in the levels of oxidative damage in the brains of tau-expressing flies compared with controls. Interestingly, Zheng et al. showed that even after an extremely strong oxidative challenge, immunohistochemical analysis only detected a very small increase in oxidized lipids in the brain proper of Drosophila (despite clear differences in the fat tissue adjacent to the brain) (39). These observations suggest a lack of sensitivity of conventional methodologies for measuring oxidative damage in the fly brain. Nevertheless, the fact that we were able to significantly rescue the neurodegenerative phenotype of tau transgenic flies by overexpression of 2 different enzymes with wellestablished antioxidant activities (Figure 3A) and by feeding with an 
A

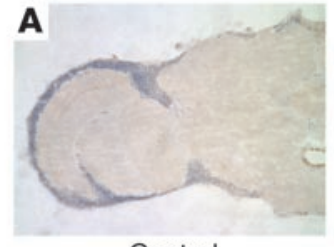
Control

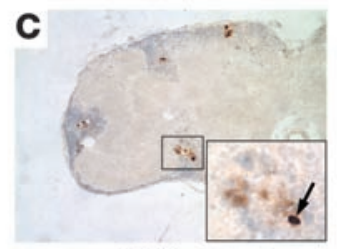

$\operatorname{tau}^{\mathrm{R} 406 \mathrm{~W}}+\operatorname{Trxr}^{\Delta 1}$
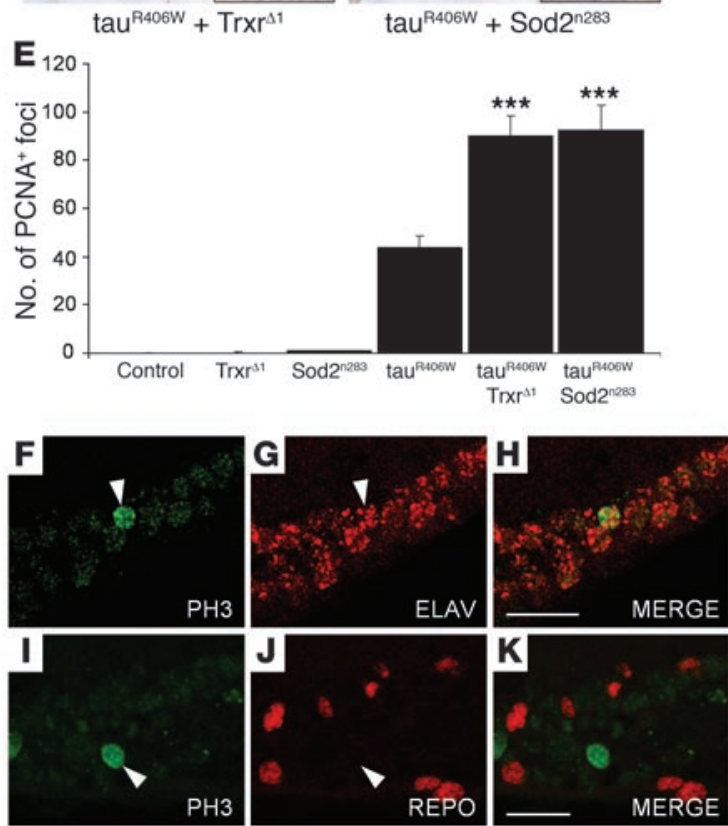

antioxidant (Figure 3B) suggests the presence of oxidative damage in our model. Whether the accumulation of oxidative damage over time is enhanced by tau overexpression remains unclear.

Although the accumulation of ROS causes oxidative stress, low to moderate levels of ROS are used under physiological conditions to regulate signaling pathways required for proper cell function. Similarly, reactive nitrogen species such as NO have oxidizing properties that can contribute to oxidative damage but also play important biological roles in processes that include neurotransmission and immune regulation (40). Overexpression of certain presenilin 1 (PS1) mutants linked to familial forms of AD in cultured neurons induces cell death that can be suppressed by NOS inhibitors (41). Consistent with the hypothesis that increased levels of NO contribute to $\mathrm{AD}$ pathogenesis, Nathan et al. reported that deletion of NOS2 in double-transgenic mice expressing mutant forms of amyloid precursor protein (APP) and PS1 protected against early mortality and decreased plaque formation (42). However, in a recent study, genetic removal of NOS2 was found to increase tau pathology and $\beta$-amyloid deposition in transgenic mice expressing mutant APP (43). These findings suggest that reducing the levels of NO can differentially affect $\mathrm{AD}$ pathology, depending on the genetic context, and may be a reflection of the dual role of reactive nitrogen species as both harmful and valuable.

\section{Figure 7}

Oxidative stress enhances cell cycle activation in tau transgenic flies. (A-D) Frontal brain sections of flies aged to day 10 were immunostained with an antibody against PCNA. PCNA-positive cells (arrows) were not detected in control flies (A) but were identified in the cortex of tau ${ }^{\text {R406W }}$. expressing flies (B). PCNA staining was increased in tau ${ }^{\mathrm{R} 406 \mathrm{~W}}$ flies heterozygous for $\operatorname{Trxr}^{\Delta 1}$ (C) and for Sod2n283 (D). Scale bar: $20 \mu \mathrm{m}$. (E) Cell cycle activation induced by expression of tau ${ }^{\mathrm{R} 406 \mathrm{~W}}$ was significantly enhanced by partial inactivation of $\operatorname{Trxr}\left({ }^{* * *} P<0.001\right)$ and Sod2 $\left.{ }^{* * *} P<0.001\right)$ activities. The number of PCNA-positive foci in the brains of 10-day-old tauR406W-expressing flies was compared with that in age-matched controls, Sod $2^{n 283}$ and $\operatorname{Tr} \mathrm{r}^{\Delta 1}$ heterozygous animals, and tauR406W transgenic flies heterozygous for Sod2 ${ }^{\text {n283 }}$ or for $\operatorname{TrXr}^{\Delta 1}$. Data points represent the mean \pm SEM. $(F-K)$ Cell cycle induction was identified in neurons. PH3 staining (green fluorescence) was found to colocalize with the neuronal cell marker elav (red fluorescence) $(\mathbf{F}-\mathbf{H})$ but not with the glial cell marker repo (red fluorescence) (I-K). Arrowheads depict the location of PH3-positive nuclei. Scale bars: $10 \mu \mathrm{m}$.

Clinical trials using antioxidants and, more specifically, vitamin E yielded conflicting results regarding the suitability of this drug for the treatment of $\mathrm{AD}$ and other neurodegenerative diseases $(31,44$, 45). Here, we show that administration of $\alpha$-tocopherol (vitamin E) significantly suppressed tau-induced neurotoxicity in Drosophila (Figure 3B). As direct comparison of drug dosages between flies and humans is not feasible, our findings provide qualitative implications for the use of vitamin $\mathrm{E}$ in the treatment of human neurodegeneration. Interestingly, similar beneficial results were recently reported by others using transgenic mouse models of human tauopathies (46) and $\mathrm{AD}(47,48)$. Taken together with these reports, our data underscore the therapeutic potential of vitamin $\mathrm{E}$.

How might increased levels of oxidative stress enhance tau neurotoxicity? Given the importance of tau phosphorylation in promoting neurotoxicity (Steinhilb et al., unpublished observations; refs. $28,29,34,35,49)$, we first determined whether oxidative damage could influence tau phosphorylation. Our analysis suggests that oxidative stress does not act by promoting tau phosphorylation at major disease-associated sites because phosphorylation of tau is not altered in contexts of exacerbated oxidative stress (Figure 5A). Additionally, increased levels of oxidative stress can significantly enhance neurodegeneration associated with a kinase insensitive form of tau that is pseudophosphorylated at 14 SP/TP sites (Figure $5 \mathrm{~B})$. These observations support a model in which oxidative damage acts downstream of tau phosphorylation to promote neurotoxicity. Alternatively, oxidative damage generated in a tau-independent manner may synergize with phosphorylated tau to enhance neurotoxicity. It is well established that oxidative damage accumulates in the aging brain and as a result of excitotoxicity in degenerative disease states $(2,50)$. Moreover, we show that tau transgenic flies are significantly more vulnerable to the free radical generator paraquat than control flies (Figure 4), indicating that tau overexpression hypersensitizes Drosophila to oxidative injury. Therefore, a time-dependent buildup of oxidative damage in the brain, caused by aging or by a pathological condition, may act in concert with phosphorylated tau to promote neurodegeneration. Determining whether oxidative stress influences other aspects of tau biochemistry, including aggregation, will require further investigation.

The exact mechanism of tau-induced neurodegeneration is still unclear. While there is no evidence of neuronal apoptosis in mouse models of tauopathy, in the Drosophila model, neuronal cell death is consistent with an apoptotic mechanism $(28,34)$. Moreover, the 
tau mutations R406W, V337M, and N279K, associated with frontotemporal dementia, were shown to enhance the vulnerability of cultured neurons to apoptotic stressors $(51,52)$. Interestingly, hallmarks of both apoptotic and necrotic cell death have been recently identified in neurons from transgenic mice expressing nonmutant forms of human tau (53). Taken together, these studies suggest that tau-induced neuronal cell death may involve both apoptotic and nonapoptotic mechanisms.

Multiple lines of evidence point to the activation of the JNK pathway as a common cellular response to oxidative injury $(16,18)$. There is also evidence of JNK activation in postmortem brain tissue derived from $\mathrm{AD}$ patients. JNK phosphorylation is elevated in the affected regions of $\mathrm{AD}$ brains but not in unaffected areas or in healthy controls $(54,55)$; this establishes a correlation between JNK activity and $\mathrm{AD}$ pathology. Interestingly, in the present study, we show that tau overexpression activates the JNK pathway in Drosophila (Figure 6). Moreover, the extent to which JNK signaling is triggered strongly correlates with the degree of toxicity generated by different tau constructs (Figure 6F). These findings are consistent with a model in which tau expression activates oxidative damage and, subsequently, the JNK pathway to promote neuronal apoptosis (16). In addition, however, tau may synergize with preexisting oxidative damage that develops during aging to promote neurodegeneration.

Abnormal activation of the cell cycle in postmitotic neurons presents a particularly attractive target for synergistic activation by tau and oxidative stress. Aberrant activation of the cell cycle machinery is a well-documented phenomenon in neurodegenerative diseases, including AD (56). Moreover, blocking cell cycle progression in various neuronal cell culture systems has been shown to protect against apoptotic cell death, suggesting that cell cycle activation may trigger apoptosis (24). Ectopic cell cycle induction has been recently associated with 3 different strains of APP-transgenic mice, in which pathology is characterized by $A \beta$ accumulation (57). Furthermore, in a recent study using our Drosophila tauopathy model, we provided compelling in vivo evidence supporting a causal role for cell cycle activation in tau-mediated apoptosis (35).

A link between oxidative injury and cell cycle activation is also well established. In a cell culture system using terminally differentiated neurons, ROS-induced DNA damage resulted in apoptosis and was accompanied by cell cycle activation (58). Oxidative stress has also been associated with the induction of cell cycle machinery in animal models of ischemia $(59,60)$. Although these studies reported cell cycle activation in models of neuronal cell death induced by an acute oxidative challenge, it remained unclear how oxidative stress affects the cell cycle during slow, progressive neurodegenerative processes. A more recent report using a mouse model of progressive ataxia has addressed this issue (61). The harlequin (Hq) mutant mouse has reduced expression of the apoptosis-inducing factor (AIF), which leads to increased levels of oxidative stress, activation of cell cycle markers, DNA replication, and progressive neurodegeneration (61). These observations strongly support a connection between oxidative injury and cell cycle activation during gradual neuronal cell death in the cerebel$\mathrm{la}$ and retinae of $\mathrm{Hq}$ mutants. In the present study, we show that genetic downregulation of antioxidant defense mechanisms in tau transgenic flies results in increased cell cycle activation (Figure 7), supporting an interaction of oxidative damage with tau-induced neurotoxicity at the level of cell cycle activation.

We demonstrate here that genetic and pharmacological manipulation of antioxidant defenses modifies tau-induced neurotox- icity in a Drosophila tauopathy model. Our results also suggest that oxidative injury enhances cell cycle activation and does not act to promote tau phosphorylation. Furthermore, we show that the JNK signaling pathway is activated in tau transgenic flies and that the levels of JNK activation correlate with the degree of tau-induced neurotoxicity. In summary, our results suggest that increased levels of oxidative stress play an active role in enhancing tau-mediated neurodegeneration, possibly through cell cycle activation, underscoring the therapeutic potential of targeting antioxidant pathways and cell cycle mechanisms for the treatment of $\mathrm{AD}$ and human tauopathies.

\section{Methods}

Drosophila stocks and genetics. Flies were grown on standard cornmeal-based Drosophila medium at $25^{\circ} \mathrm{C}$. The UAS-tan WT, UAS-tan ${ }^{R 406 W}$, and UAS-tan ${ }^{E 14}$ transgenic lines were previously described $(27,35)$. Panneuronal transgene expression was achieved using the elav-GAL4 driver. Drosophila lines expressing equivalent levels of tau transgenes in the brain were used. Two lines of tau ${ }^{\mathrm{E} 14}$-expressing flies were used, line 22 (Figure 5) and line 26 (Figure 6). In tau ${ }^{\mathrm{E} 14(26)}$ flies, tau expression levels matched those of $\operatorname{tau}^{\mathrm{WT}}$ and $\operatorname{tau}^{\mathrm{R} 406 \mathrm{~W}}$ transgenic flies used in this study. The tau ${ }^{\text {E14(22) }}$ transgenic flies expressed slightly higher levels of tau than tau ${ }^{\text {E14(26) }}$ transgenic flies, which resulted in slightly higher levels of neurotoxicity.

Drug feeding. For vitamin E feeding (Figure 3), Drosophila were fed 0.5 $\mathrm{mM}$ or $1.5 \mathrm{mM}$ of $\alpha$-tocopherol (Sigma-Aldrich) that had been dissolved in soybean oil and mixed with standard cornmeal medium. Control flies grown in the absence of drug were fed standard cornmeal medium with $3 \%$ soybean oil solvent alone. Drug and control feeding trials were performed for 10 days after eclosion. Flies were transferred to fresh food every second day. For paraquat feeding (Figure 4), flies were fed $30 \mathrm{mM}$ of methyl viologen (Sigma-Aldrich) in instant Drosophila medium (Carolina). Control flies were fed instant medium only. One-day-old adult flies were kept in $30 \mathrm{mM}$ paraquat or in drug-free medium for 48 hours after eclosion.

Histology, immunostaining, and TUNEL analysis. Heads from adult flies fixed in formalin at 5 or 10 days after eclosion were embedded in paraffin and sectioned in a frontal orientation. Serial $4-\mu \mathrm{m}$ sections spanning the whole brain were placed on a glass slide and subject to further analysis. Brain morphology was evaluated by staining paraffin sections with $\mathrm{H} \& \mathrm{E}$ according to standard protocols.

For immunostaining analysis on paraffin sections, antigen retrieval was achieved by boiling ( 15 minutes, $10 \mathrm{mM}$ sodium citrate, $\mathrm{pH}$ 6.6). Immunostaining was performed using an avidin-biotin-peroxidase complex (ABC) method (VECTASTAIN ABC Kit; Vector Laboratories) or secondary antibodies coupled to Alexa Fluor 488 or Alexa Fluor 555 (Invitrogen). Primary antibodies included anti-active JNK (Promega; $1: 300)$, anti- $\beta$-gal (Promega; 1:500), anti-elav (Developmental Studies Hybridoma Bank; 1:200), anti-PCNA (Biomeda; 1:500), anti-PH3 (Upstate USA Inc.; 1:500), and anti-repo (Developmental Studies Hybridoma Bank; 1:250). The appropriate secondary antibodies were diluted 1:200, and histochemical detection was done by developing with DAB (Sigma-Aldrich). Nuclei were counterstained with hematoxylin.

Apoptotic cell death was detected by TUNEL staining on paraffin sections according to the manufacturer's instructions (TdT FragEL DNA fragmentation kit; Calbiochem; EMD Biosciences). Quantification of TUNEL-positive cells (Figures 2, 3, 5, and 6 and Supplemental Figure 2), $\beta$-gal-positive nuclei (Figure 6), PCNA-positive foci (Figure 7), and vacuoles with diameter greater than $3 \mu \mathrm{m}$ (Figure 1 and Supplemental Figure 2 ) was performed by visually examining serial frontal sections $(4 \mu \mathrm{m}$ thick) spanning the entire fly brain. All positive occurrences identified in each hemibrain in the regions of the central body, the lobula, and the 
medulla were manually scored (anatomic information on the fly brain can be found at Basic Atlas of the Drosophila Brain, http://web.neurobio. arizona.edu/Flybrain/html/atlas/index.html). In all graphs, each data point represents the average number of counts obtained for at least 8 hemibrains. The number of hemibrains used in each experiment was sufficient to account for the degree of variability among different samples and to yield highly significant results, noted by asterisks. Statistical analysis was performed using 1-way ANOVA with Student-Newman-Keuls post hoc test, and data points represent the mean \pm SEM. Results were considered significant at $P<0.05$.

Western blot. Heads from adult flies aged for 10 days after eclosion were homogenized in $2 \times$ Laemmli buffer, resolved by SDS-PAGE, and subject to immunoblotting analysis using standard procedures. Primary antibodies and their respective dilutions were as follows: anti-AT8 (Pierce Biotechnology; 1:50,000), anti-AT100 (Pierce Biotechnology; 1:500), anti-AT180 (Innogenetics; 1:2,000), anti-TG3 (P. Davies, Albert Einstein College of Medicine, Bronx, New York, USA; 1:1,000), anti-PHF1 (P. Davies; 1:50,000), anti-Tau1 (Chemicon International; 1:2,000), anti-total tau (Dako; 1:1,000,000), and anti-actin (Sigma-Aldrich; 1:50,000). Prior to immunostaining with AT8 and TG3 antibodies, nitrocellulose membranes were boiled (5 minutes, $1 \times$ PBS) for antigen retrieval. HRP-conjugated secondary antibodies were used at a 1:50,000 dilution, and signal detection was performed with chemiluminescence (Pierce Biotechnology).

\section{Acknowledgments}

We thank F. Missirlis, H. Jackle, J. Phillips, H. Jasper, D. Bohmann, and the Drosophila stock center for providing fly lines; J. Locascio for statistical consultation; and V. Khurana for critical comments on the manuscript. TG3 and PHF1 monoclonal antibodies were kind gifts of P. Davies. This work was supported by NIH grants AG19790 and AG005134 (to M.B. Feany) and by a grant from the Portuguese Foundation for Science and Technology (FCT reference: SFRH/BPD/20685/2004; to D. Dias-Santagata).

Received for publication April 7, 2006, and accepted in revised form October 31, 2006.

Address correspondence to: Mel B. Feany, Department of Pathology, Brigham and Women's Hospital and Harvard Medical School, Harvard New Research Building, Room 652, 77 Avenue Louis Pasteur, Boston, Massachusetts 02115, USA. Phone: (617) 525-4405; Fax: (617) 525-4422; E-mail: mel_feany@hms.harvard.edu.
1. Cummings, J.L. 2004. Alzheimer's disease. N. Engl. J. Med. 351:56-67.

2. Sohal, R.S. 2002. Oxidative stress hypothesis of aging. Free Radic. Biol. Med. 33:573-574.

3. Martindale, J.L., and Holbrook, N.J. 2002. Cellular response to oxidative stress: signaling for suicide and survival. J. Cell. Physiol. 192:1-15.

4. Duttaroy, A., Paul, A., Kundu, M., and Belton, A. 2003. A Sod2 null mutation confers severely reduced adult life span in Drosophila. Genetics. 165:2295-2299.

5. Kirby, K., Hu, J., Hilliker, A.J., and Phillips, J.P. 2002. RNA interference-mediated silencing of Sod 2 in Drosophila leads to early adult-onset mortality and elevated endogenous oxidative stress. Proc. Natl. Acad. Sci. U. S. A. 99:16162-16167.

6. Griswold, C.M., Matthews, A.L., Bewley, K.E., and Mahaffey, J.W. 1993. Molecular characterization and rescue of acatalasemic mutants of Drosophila melanogaster. Genetics. 134:781-788.

7. Phillips, J.P., Campbell, S.D., Michaud, D., Charbonneau, M., and Hilliker, A.J. 1989. Null mutation of copper/zinc superoxide dismutase in Drosophila confers hypersensitivity to paraquat and reduced longevity. Proc. Natl. Acad. Sci. U. S. A. 86:2761-2765.

8. Sun, J., Folk, D., Bradley, T.J., and Tower, J. 2002. Induced overexpression of mitochondrial Mnsuperoxide dismutase extends the life span of adult Drosophila melanogaster. Genetics. 161:661-672.

9. Schriner, S.E., et al. 2005. Extension of murine life span by overexpression of catalase targeted to mitochondria. Science. 308:1909-1911.

10. Orr, W.C., and Sohal, R.S. 1994. Extension of lifespan by overexpression of superoxide dismutase and catalase in Drosophila melanogaster. Science. 263:1128-1130.

11. Parkes, T.L., et al. 1998. Extension of Drosophila lifespan by overexpression of human SOD1 in motorneurons. Nat. Genet. 19:171-174.

12. Rhee, S.G., Chae, H.Z., and Kim, K. 2005. Peroxiredoxins: a historical overview and speculative preview of novel mechanisms and emerging concepts in cell signaling. Free Radic. Biol. Med. 38:1543-1552.

13. Missirlis, F., et al. 2002. Mitochondrial and cytoplasmic thioredoxin reductase variants encoded by a single Drosophila gene are both essential for viability. J. Biol. Chem. 277:11521-11526.

14. Missirlis, F., et al. 2003. A putative glutathione peroxidase of Drosophila encodes a thioredoxin peroxidase that provides resistance against oxidative stress but fails to complement a lack of catalase activity. Biol. Chem. 384:463-472.

15. Missirlis, F., Phillips, J.P., and Jackle, H. 2001. Cooperative action of antioxidant defense systems in Drosophila. Curr. Biol. 11:1272-1277.

16. Matsuzawa, A., and Ichijo, H. 2005. Stress-responsive protein kinases in redox-regulated apoptosis signaling. Antioxid. Redox Signal. 7:472-481.

17. Andersen, J.K. 2004. Oxidative stress in neurodegeneration: cause or consequence? Nat. Med. 10(Suppl.):S18-S25.

18. Zhu, X., et al. 2004. Oxidative stress signalling in Alzheimer's disease. Brain Res. 1000:32-39.

19. Markesbery, W.R., and Lovell, M.A. 1998. Fourhydroxynonenal, a product of lipid peroxidation, is increased in the brain in Alzheimer's disease. Neurobiol. Aging. 19:33-36.

20. Smith, M.A., Richey Harris, P.L., Sayre, L.M., Beckman, J.S., and Perry, G. 1997. Widespread peroxynitrite-mediated damage in Alzheimer's disease. J. Neurosci. 17:2653-2657.

21. Ogawa, O., et al. 2003. Ectopic localization of phosphorylated histone $\mathrm{H} 3$ in Alzheimer's disease: a mitotic catastrophe? Acta Neuropathol. (Berl.). 105:524-528.

22. Busser, J., Geldmacher, D.S., and Herrup, K. 1998. Ectopic cell cycle proteins predict the sites of neuronal cell death in Alzheimer's disease brain. J. Neurosci. 18:2801-2807.

23. Husseman, J.W., Nochlin, D., and Vincent, I. 2000. Mitotic activation: a convergent mechanism for a cohort of neurodegenerative diseases. Neurobiol. Aging. 21:815-828.

24. Langley, B., and Ratan, R.R. 2004. Oxidative stressinduced death in the nervous system: cell cycle dependent or independent? J. Neurosci. Res. 77:621-629.

25. Lee, V.M., Goedert, M., and Trojanowski, J.Q. 2001. Neurodegenerative tauopathies. Annu. Rev. Neurosci. 24:1121-1159.

26. Hutton, M. 2000. Molecular genetics of chromosome 17 tauopathies. Ann. N. Y. Acad. Sci. 920:63-73.

27. Wittmann, C.W., et al. 2001. Tauopathy in Drosophila: neurodegeneration without neurofibrillary tangles. Science. 293:711-714.

28. Jackson, G.R., et al. 2002. Human wild-type tau interacts with wingless pathway components and produces neurofibrillary pathology in Drosophila. Neuron. 34:509-519.

29. Nishimura, I., Yang, Y., and Lu, B. 2004. PAR-1 kinase plays an initiator role in a temporally ordered phosphorylation process that confers tau toxicity in Drosophila. Cell. 116:671-682.

30. Missirlis, F., et al. 2003. Compartment-specific protection of iron-sulfur proteins by superoxide dismutase. J. Biol. Chem. 278:47365-47369.

31. Tucker, J.M., and Townsend, D.M. 2005. Alphatocopherol: roles in prevention and therapy of human disease. Biomed. Pharmacother. 59:380-387.

32. Arking, R., Buck, S., Berrios, A., Dwyer, S., and Baker, G.T., 3rd. 1991. Elevated paraquat resistance can be used as a bioassay for longevity in a genetically based long-lived strain of Drosophila. Dev. Genet. 12:362-370.

33. Yen, S.H., et al. 1995. Alzheimer neurofibrillary lesions: molecular nature and potential roles of different components. Neurobiol. Aging. 16:381-387.

34. Shulman, J.M., and Feany, M.B. 2003. Genetic modifiers of tauopathy in Drosophila. Genetics. 165:1233-1242.

35. Khurana, V., et al. 2006. TOR-mediated cell-cycle activation causes neurodegeneration in a Drosophila tauopathy model. Curr. Biol. 16:230-241.

36. Zhu, X., et al. 2003. Oxidative stress and neuronal adaptation in Alzheimer disease: the role of SAPK pathways. Antioxid. Redox Signal. 5:571-576.

37. Martin-Blanco, E., et al. 1998. puckered encodes a phosphatase that mediates a feedback loop regulating JNK activity during dorsal closure in Drosophila. Genes Dev. 12:557-570.

38. Nunomura, A., et al. 1999. Neuronal RNA oxidation in Alzheimer's disease and Down's syndrome. Ann. N. Y. Acad. Sci. 893:362-364.

39. Zheng, J., Mutcherson, R., 2nd, and Helfand, S.L. 2005. Calorie restriction delays lipid oxidative damage in Drosophila melanogaster. Aging Cell. 4:209-216.

40. Valko, M., Leibfritz, D., Moncol, J., Cronin, M.T., Mazur, M., and Telser, J. 2006. Free radicals and antioxidants in normal physiological functions and human disease. Int. J. Biochem. Cell. Biol. 39:44-84.

41. Hashimoto, Y., et al. 2004. Amino- and carboxyl-terminal mutants of presenilin 1 cause neuronal cell death through distinct toxic mechanisms: study of 27 different presenilin 1 mutants. J. Neurosci. Res. 75:417-428.

42. Nathan, C., et al. 2005. Protection from Alzheimer's-like disease in the mouse by genetic ablation of inducible nitric oxide synthase. J. Exp. Med. 202:1163-1169. 
43. Colton, C.A., et al. 2006. NO synthase 2 (NOS2) deletion promotes multiple pathologies in a mouse model of Alzheimer's disease. Proc. Natl. Acad. Sci. U. S. A. 103:12867-12872.

44. Boothby, L.A., and Doering, P.L. 2005. Vitamin C and vitamin E for Alzheimer's disease. Ann. Phar macother. 39:2073-2080.

45. Kontush, K., and Schekatolina, S. 2004. Vitamin $\mathrm{E}$ in neurodegenerative disorders: Alzheimer's disease. Ann. N. Y. Acad. Sci. 1031:249-262.

46. Nakashima, H., et al. 2004. Effects of alpha-tocopherol on an animal model of tauopathies. Free Radic. Biol. Med. 37:176-186.

47. Sung, S., et al. 2004. Early vitamin E supplementation in young but not aged mice reduces Abeta levels and amyloid deposition in a transgenic model of Alzheimer's disease. FASEB J. 18:323-325.

48. Conte, V., et al. 2004. Vitamin E reduces amyloidosis and improves cognitive function in $\operatorname{Tg} 2576$ mice following repetitive concussive brain injury. J. Neurochem. 90:758-764.

49. Stoothoff, W.H., and Johnson, G.V. 2005. Tau phos- phorylation: physiological and pathological consequences. Biochim. Biophys. Acta. 1739:280-297.

50. Mattson, M.P. 2003. Excitotoxic and excitoprotective mechanisms: abundant targets for the prevention and treatment of neurodegenerative disorders. Neuromolecular Med. 3:65-94.

51. Krishnamurthy, P.K., and Johnson, G.V. 2004 Mutant (R406W) human tau is hyperphosphorylated and does not efficiently bind microtubules in a neuronal cortical cell model. J. Biol. Chem. 279:7893-7900.

52. Furukawa, K., et al. 2000. Pro-apoptotic effects of tau mutations in chromosome 17 frontotemporal dementia and parkinsonism. Neuroreport. 11:57-60.

53. Andorfer, C., et al. 2005. Cell-cycle reentry and cell death in transgenic mice expressing nonmutant human tau isoforms. J. Neurosci. 25:5446-5454.

54. Zhu, X., et al. 2001. Activation and redistribution of c-jun $\mathrm{N}$-terminal kinase/stress activated protein kinase in degenerating neurons in Alzheimer's disease. J. Neurochem. 76:435-441.

55. Shoji, M., et al. 2000. JNK activation is associated with intracellular beta-amyloid accumulation. Brain Res. Mol. Brain Res. 85:221-233.

56. Herrup, K., Neve, R., Ackerman, S.L., and Copani, A. 2004. Divide and die: cell cycle events as triggers of nerve cell death. J. Neurosci. 24:9232-9239.

57. Yang, Y., Varvel, N.H., Lamb, B.T., and Herrup, K. 2006. Ectopic cell cycle events link human Alzheimer's disease and amyloid precursor protein transgenic mouse models. J. Neurosci. 26:775-784.

58. Kruman, I.I., et al. 2004. Cell cycle activation linked to neuronal cell death initiated by DNA damage. Neuron. 41:549-561.

59. Guegan, C., Levy, V., David, J.P., Ajchenbaum-Cymbalista, F., and Sola, B. 1997. c-Jun and cyclin D1 proteins as mediators of neuronal death after a focal ischaemic insult. Neuroreport. 8:1003-1007.

60. Li, Y., Chopp, M., Powers, C., and Jiang, N. 1997. Apoptosis and protein expression after focal cerebral ischemia in rat. Brain Res. 765:301-312.

61. Klein, J.A., et al. 2002. The harlequin mouse mutation downregulates apoptosis-inducing factor. Nature. 419:367-374. 